\title{
Longitudinal double-spin asymmetry and cross section for inclusive neutral pion production at midrapidity in polarized proton collisions at $\sqrt{s}=200 \mathrm{GeV}$
}

B. I. Abelev, ${ }^{8}$ M. M. Aggarwal, ${ }^{30}$ Z. Ahammed,${ }^{47}$ A. V. Alakhverdyants, ${ }^{17}$ B. D. Anderson, ${ }^{18}$ D. Arkhipkin, ${ }^{3}$ G. S. Averichev, ${ }^{17}$ J. Balewski, ${ }^{22}$ O. Barannikova, ${ }^{8}$ L. S. Barnby, ${ }^{2}$ S. Baumgart,${ }^{52}$ D. R. Beavis,${ }^{3}$ R. Bellwied,${ }^{50}$ F. Benedosso, ${ }^{27}$ M. J. Betancourt,${ }^{22}$ R. R. Betts, ${ }^{8}$ A. Bhasin, ${ }^{16}$ A. K. Bhati, ${ }^{30}$ H. Bichsel, ${ }^{49}$ J. Bielcik, ${ }^{10}$ J. Bielcikova, ${ }^{11}$ B. Biritz, ${ }^{6}$ L. C. Bland, ${ }^{3}$ B. E. Bonner,${ }^{36}$ J. Bouchet,${ }^{18}$ E. Braidot,${ }^{27}$ A. V. Brandin, ${ }^{25}$ A. Bridgeman, ${ }^{1}$ E. Bruna, ${ }^{52}$ S. Bueltmann, ${ }^{29}$ I. Bunzarov,${ }^{17}$ T. P. Burton,${ }^{2}$ X. Z. Cai,${ }^{40}$ H. Caines,${ }^{52}$ M. Calderón de la Barca Sánchez,${ }^{5}$ O. Catu, ${ }^{52}$ D. Cebra,${ }^{5}$ R. Cendejas, ${ }^{6}$ M. C. Cervantes,${ }^{42}$ Z. Chajecki, ${ }^{28}$ P. Chaloupka, ${ }^{11}$ S. Chattopadhyay ${ }^{47}$ H. F. Chen, ${ }^{38}$ J. H. Chen, ${ }^{40}$ J. Y. Chen, ${ }^{51}$ J. Cheng, ${ }^{44}$ M. Cherney ${ }^{9}$ A. Chikanian, ${ }^{52}$ K. E. Choi,${ }^{34}$ W. Christie, ${ }^{3}$ P. Chung, ${ }^{11}$ R. F. Clarke,${ }^{42}$ M. J. M. Codrington, ${ }^{42}$ R. Corliss, ${ }^{22}$ J. G. Cramer ${ }^{49}$ H. J. Crawford,${ }^{4}$ D. Das, ${ }^{5}$ S. Dash, ${ }^{13}$ A. Davila Leyva, ${ }^{43}$ L. C. De Silva,${ }^{50}$ R. R. Debbe, ${ }^{3}$ T. G. Dedovich, ${ }^{17}$ M. DePhillips, ${ }^{3}$ A. A. Derevschikov ${ }^{32}$ R. Derradi de Souza,${ }^{7}$ L. Didenko, ${ }^{3}$ P. Djawotho, ${ }^{42}$

S. M. Dogra,${ }^{16}$ X. Dong, ${ }^{21}$ J. L. Drachenberg, ${ }^{42}$ J. E. Draper, ${ }^{5}$ J. C. Dunlop,${ }^{3}$ M. R. Dutta Mazumdar, ${ }^{47}$ L. G. Efimov, ${ }^{17}$ E. Elhalhuli, ${ }^{2}$ M. Elnimr,${ }^{50}$ J. Engelage,${ }^{4}$ G. Eppley,${ }^{36}$ B. Erazmus, ${ }^{41}$ M. Estienne,${ }^{41}$ L. Eun, ${ }^{31}$ P. Fachini,${ }^{3}$ R. Fatemi, ${ }^{19}$ J. Fedorisin, ${ }^{17}$ R. G. Fersch, ${ }^{19}$ P. Filip,${ }^{17}$ E. Finch,${ }^{52}$ V. Fine,${ }^{3}$ Y. Fisyak, ${ }^{3}$ C. A. Gagliardi,${ }^{42}$ D. R. Gangadharan, ${ }^{6}$ M. S. Ganti, ${ }^{47}$ E. J. Garcia-Solis, ${ }^{8}$ A. Geromitsos,${ }^{41}$ F. Geurts, ${ }^{36}$ V. Ghazikhanian, ${ }^{6}$ P. Ghosh,${ }^{47}$ Y. N. Gorbunov ${ }^{9}$ A. Gordon,${ }^{3}$ O. Grebenyuk, ${ }^{21}$ D. Grosnick, ${ }^{46}$ B. Grube,${ }^{34}$ S. M. Guertin, ${ }^{6}$ A. Gupta,${ }^{16}$ N. Gupta,${ }^{16}$ W. Guryn ${ }^{3}$ B. Haag, ${ }^{5}$ T. J. Hallman,${ }^{3}$ A. Hamed, ${ }^{42}$ L-X. Han ${ }^{40}$ J. W. Harris,${ }^{52}$ J. P. Hays-Wehle,${ }^{22}$ M. Heinz,${ }^{52}$

S. Heppelmann, ${ }^{31}$ A. Hirsch, ${ }^{33}$ E. Hjort, ${ }^{21}$ A. M. Hoffman, ${ }^{22}$ G. W. Hoffmann, ${ }^{43}$ D. J. Hofman, ${ }^{8}$ R. S. Hollis, ${ }^{8}$ H. Z. Huang, ${ }^{6}$ T. J. Humanic, ${ }^{28}$ L. Huo,${ }^{42}$ G. Igo, ${ }^{6}$ A. Iordanova,${ }^{8}$ P. Jacobs,${ }^{21}$ W. W. Jacobs, ${ }^{15}$ P. Jakl, ${ }^{11}$ C. Jena, ${ }^{13}$

F. Jin,${ }^{40}$ C. L. Jones, ${ }^{22}$ P. G. Jones, ${ }^{2}$ J. Joseph,${ }^{18}$ E. G. Judd,${ }^{4}$ S. Kabana,${ }^{41}$ K. Kajimoto, ${ }^{43}$ K. Kang, ${ }^{44}$

J. Kapitan, ${ }^{11}$ K. Kauder, ${ }^{8}$ D. Keane,${ }^{18}$ A. Kechechyan, ${ }^{17}$ D. Kettler ${ }^{49}$ D. P. Kikola, ${ }^{21}$ J. Kiryluk, ${ }^{21}$ A. Kisiel ${ }^{48}$

S. R. Klein, ${ }^{21}$ A. G. Knospe, ${ }^{52}$ A. Kocoloski, ${ }^{22}$ D. D. Koetke,${ }^{46}$ T. Kollegger, ${ }^{12}$ J. Konzer, ${ }^{33}$ M. Kopytine,${ }^{18}$

I. Koralt, ${ }^{29}$ W. Korsch, ${ }^{19}$ L. Kotchenda, ${ }^{25}$ V. Kouchpil,${ }^{11}$ P. Kravtsov ${ }^{25}$ K. Krueger, ${ }^{1}$ M. Krus,${ }^{10}$ L. Kumar, ${ }^{30}$

P. Kurnadi, ${ }^{6}$ M. A. C. Lamont, ${ }^{3}$ J. M. Landgraf, ${ }^{3}$ S. LaPointe, ${ }^{50}$ J. Lauret,${ }^{3}$ A. Lebedev,${ }^{3}$ R. Lednicky ${ }^{17}$ C-H. Lee ${ }^{34}$ J. H. Lee ${ }^{3}$ W. Leight,${ }^{22}$ M. J. LeVine, ${ }^{3}$ C. Li ${ }^{38}$ L. Li, ${ }^{43}$ N. Li,${ }^{51}$ W. Li,${ }^{40}$ X. Li,${ }^{33}$ X. Li,${ }^{39}$ Y. Li,${ }^{44}$ Z. Li, ${ }^{51}$ G. Lin, ${ }^{52}$ S. J. Lindenbaum,${ }^{26}$ M. A. Lisa, ${ }^{28}$ F. Liu, ${ }^{51}$ H. Liu,${ }^{5}$ J. Liu, ${ }^{36}$ T. Ljubicic, ${ }^{3}$ W. J. Llope, ${ }^{36}$ R. S. Longacre ${ }^{3}$ W. A. Love,${ }^{3}$ Y. Lu,${ }^{38}$ G. L. Ma ${ }^{40}$ Y. G. Ma ${ }^{40}$ D. P. Mahapatra, ${ }^{13}$ R. Majka, ${ }^{52}$ O. I. Mall, ${ }^{5}$ L. K. Mangotra, ${ }^{16}$ R. Manweiler, ${ }^{46}$ S. Margetis, ${ }^{18}$ C. Markert, ${ }^{43}$ H. Masui, ${ }^{21}$ H. S. Matis ${ }^{21}$ Yu. A. Matulenko, ${ }^{32}$ D. McDonald ${ }^{36}$ T. S. McShane,${ }^{9}$ A. Meschanin, ${ }^{32}$ R. Milner ${ }^{22}$ N. G. Minaev ${ }^{32}$ S. Mioduszewski, ${ }^{42}$ A. Mischke, ${ }^{27}$ M. K. Mitrovski, ${ }^{12}$ B. Mohanty, ${ }^{47}$ M. M. Mondal, ${ }^{47}$ D. A. Morozov, ${ }^{32}$ M. G. Munhoz,${ }^{37}$ B. K. Nandi, ${ }^{14}$

C. Nattrass, ${ }^{52}$ T. K. Nayak, ${ }^{47}$ J. M. Nelson, ${ }^{2}$ P. K. Netrakanti, ${ }^{33}$ M. J. Ng, ${ }^{4}$ L. V. Nogach, ${ }^{32}$ S. B. Nurushev ${ }^{32}$ G. Odyniec ${ }^{21}$ A. Ogawa, ${ }^{3}$ H. Okada,${ }^{3}$ V. Okorokov ${ }^{25}$ D. Olson, ${ }^{21}$ M. Pachr,${ }^{10}$ B. S. Page, ${ }^{15}$ S. K. Pal, ${ }^{47}$

Y. Pandit, ${ }^{18}$ Y. Panebratsev,${ }^{17}$ T. Pawlak, ${ }^{48}$ T. Peitzmann, ${ }^{27}$ V. Perevoztchikov, ${ }^{3}$ C. Perkins, ${ }^{4}$ W. Peryt,${ }^{48}$ S. C. Phatak, ${ }^{13}$ P. Pile,${ }^{3}$ M. Planinic, ${ }^{53}$ M. A. Ploskon, ${ }^{21}$ J. Pluta, ${ }^{48}$ D. Plyku, ${ }^{29}$ N. Poljak, ${ }^{53}$ A. M. Poskanzer, ${ }^{21}$

B. V. K. S. Potukuchi, ${ }^{16}$ C. B. Powell,${ }^{21}$ D. Prindle, ${ }^{49}$ C. Pruneau, ${ }^{50}$ N. K. Pruthi, ${ }^{30}$ P. R. Pujahari, ${ }^{14}$ J. Putschke, ${ }^{52}$ R. Raniwala, ${ }^{35}$ S. Raniwala, ${ }^{35}$ R. L. Ray, ${ }^{43}$ R. Redwine, ${ }^{22}$ R. Reed, ${ }^{5}$ J. M. Rehberg, ${ }^{12}$ H. G. Ritter, ${ }^{21}$ J. B. Roberts ${ }^{36}$ O. V. Rogachevskiy, ${ }^{17}$ J. L. Romero, ${ }^{5}$ A. Rose,${ }^{21}$ C. Roy,${ }^{41}$ L. Ruan,${ }^{3}$ M. J. Russcher, ${ }^{27}$ R. Sahoo, ${ }^{41}$ S. Sakai,${ }^{6}$ I. Sakrejda, ${ }^{21}$ T. Sakuma,${ }^{22}$ S. Salur,${ }^{5}$ J. Sandweiss, ${ }^{52}$ E. Sangaline, ${ }^{5}$ J. Schambach,${ }^{43}$ R. P. Scharenberg, ${ }^{33}$ N. Schmitz, ${ }^{23}$ T. R. Schuster, ${ }^{12}$ J. Seele, ${ }^{22}$ J. Seger,${ }^{9}$ I. Selyuzhenkov, ${ }^{15}$ P. Seyboth, ${ }^{23}$ E. Shahaliev, ${ }^{17}$ M. Shao,${ }^{38}$ M. Sharma,${ }^{50}$ S. S. Shi,${ }^{51}$ E. P. Sichtermann, ${ }^{21}$ F. Simon,${ }^{23}$ R. N. Singaraju ${ }^{47}$ M. J. Skoby ${ }^{33}$ N. Smirnov, ${ }^{52}$ P. Sorensen, ${ }^{3}$ J. Sowinski, ${ }^{15}$ H. M. Spinka, ${ }^{1}$ B. Srivastava, ${ }^{33}$ T. D. S. Stanislaus, ${ }^{46}$ D. Staszak, ${ }^{6}$ J. R. Stevens, ${ }^{15}$ R. Stock, ${ }^{12}$ M. Strikhanov, ${ }^{25}$ B. Stringfellow, ${ }^{33}$ A. A. P. Suaide, ${ }^{37}$ M. C. Suarez, ${ }^{8}$ N. L. Subba, ${ }^{18}$ M. Sumbera, ${ }^{11}$ X. M. Sun, ${ }^{21}$ Y. Sun, ${ }^{38}$ Z. Sun, ${ }^{20}$ B. Surrow,${ }^{22}$ T. J. M. Symons, ${ }^{21}$ A. Szanto de Toledo, ${ }^{37}$ J. Takahashi, ${ }^{7}$ A. H. Tang, ${ }^{3}$ Z. Tang, ${ }^{38}$ L. H. Tarini,${ }^{50}$ T. Tarnowsky, ${ }^{24}$ D. Thein, ${ }^{43}$ J. H. Thomas, ${ }^{21}$ J. Tian, ${ }^{40}$ A. R. Timmins,${ }^{50}$ S. Timoshenko, ${ }^{25}$ D. Tlusty,${ }^{11}$ M. Tokarev ${ }^{17}$ T. A. Trainor,${ }^{49}$ V. N. Tram,${ }^{21}$ S. Trentalange,${ }^{6}$ R. E. Tribble, ${ }^{42}$ O. D. Tsai,${ }^{6}$ J. Ulery, ${ }^{33}$ T. Ullrich,${ }^{3}$ D. G. Underwood,${ }^{1}$ G. Van Buren, ${ }^{3}$ G. van Nieuwenhuizen, ${ }^{22}$ J. A. Vanfossen, Jr., ${ }^{18}$ R. Varma,${ }^{14}$ G. M. S. Vasconcelos, ${ }^{7}$ A. N. Vasiliev, ${ }^{32}$ F. Videbaek, ${ }^{3}$ Y. P. Viyogi, ${ }^{47}$ S. Vokal, ${ }^{17}$ S. A. Voloshin, ${ }^{50}$ M. Wada,${ }^{43}$ M. Walker, ${ }^{22}$ F. Wang, ${ }^{33}$ G. Wang, ${ }^{6}$

H. Wang, ${ }^{24}$ J. S. Wang, ${ }^{20}$ Q. Wang, ${ }^{33}$ X. Wang, ${ }^{44}$ X. L. Wang, ${ }^{38}$ Y. Wang, ${ }^{44}$ G. Webb, ${ }^{19}$ J. C. Webb, ${ }^{46}$ G. D. Westfall, ${ }^{24}$ C. Whitten Jr. ${ }^{6}$ H. Wieman, ${ }^{21}$ E. Wingfield, ${ }^{43}$ S. W. Wissink, ${ }^{15}$ R. Witt, ${ }^{45}$ Y. Wu, ${ }^{51}$ W. Xie, ${ }^{33}$ N. Xu, ${ }^{21}$ Q. H. Xu ${ }^{39}$ W. Xu ${ }^{6}$ Y. Xu, ${ }^{38}$ Z. Xu, ${ }^{3}$ L. Xue, ${ }^{40}$ Y. Yang, ${ }^{20}$ P. Yepes,${ }^{36}$ K. Yip,${ }^{3}$ I-K. Yoo, ${ }^{34}$ Q. Yue,${ }^{44}$ M. Zawisza, ${ }^{48}$ H. Zbroszczyk, ${ }^{48}$ W. Zhan, ${ }^{20}$ S. Zhang, ${ }^{40}$ W. M. Zhang, ${ }^{18}$ X. P. Zhang, ${ }^{21}$ Y. Zhang, ${ }^{21}$ Z. P. Zhang, ${ }^{38}$ J. Zhao ${ }^{40}$ C. Zhong ${ }^{40}$ J. Zhou, ${ }^{36}$ W. Zhou, ${ }^{39}$ X. Zhu ${ }^{44}$ Y. H. Zhu ${ }^{40}$ R. Zoulkarneev, ${ }^{17}$ and Y. Zoulkarneeva ${ }^{17}$ 
${ }^{1}$ Argonne National Laboratory, Argonne, Illinois 60439, USA

${ }^{2}$ University of Birmingham, Birmingham, United Kingdom

${ }^{3}$ Brookhaven National Laboratory, Upton, New York 11973, USA

${ }^{4}$ University of California, Berkeley, California 94720, USA

${ }^{5}$ University of California, Davis, California 95616, USA

${ }^{6}$ University of California, Los Angeles, California 90095, USA

${ }^{7}$ Universidade Estadual de Campinas, Sao Paulo, Brazil

${ }^{8}$ University of Illinois at Chicago, Chicago, Illinois 60607, USA

${ }^{9}$ Creighton University, Omaha, Nebraska 68178, USA

${ }^{10}$ Czech Technical University in Prague, FNSPE, Prague, 115 19, Czech Republic

${ }^{11}$ Nuclear Physics Institute AS CR, 25068 Rež/Prague, Czech Republic ${ }^{12}$ University of Frankfurt, Frankfurt, Germany

${ }^{13}$ Institute of Physics, Bhubaneswar 751005, India

${ }^{14}$ Indian Institute of Technology, Mumbai, India

${ }^{15}$ Indiana University, Bloomington, Indiana 47408, USA

${ }^{16}$ University of Jammu, Jammu 180001, India

${ }^{17}$ Joint Institute for Nuclear Research, Dubna, 141 980, Russia

${ }^{18}$ Kent State University, Kent, Ohio 44242, USA

${ }^{19}$ University of Kentucky, Lexington, Kentucky, 40506-0055, USA

${ }^{20}$ Institute of Modern Physics, Lanzhou, China

${ }^{21}$ Lawrence Berkeley National Laboratory, Berkeley, California 94720, USA

${ }^{22}$ Massachusetts Institute of Technology, Cambridge, MA 02139-4307, USA

${ }^{23}$ Max-Planck-Institut für Physik, Munich, Germany

${ }^{24}$ Michigan State University, East Lansing, Michigan 48824, USA

${ }^{25}$ Moscow Engineering Physics Institute, Moscow Russia

${ }^{26}$ City College of New York, New York City, New York 10031, USA

${ }^{27}$ NIKHEF and Utrecht University, Amsterdam, The Netherlands

${ }^{28}$ Ohio State University, Columbus, Ohio 43210, USA

${ }^{29}$ Old Dominion University, Norfolk, VA, 23529, USA

${ }^{30}$ Panjab University, Chandigarh 160014, India

${ }^{31}$ Pennsylvania State University, University Park, Pennsylvania 16802, USA

${ }^{32}$ Institute of High Energy Physics, Protvino, Russia

${ }^{33}$ Purdue University, West Lafayette, Indiana 47907, USA

${ }^{34}$ Pusan National University, Pusan, Republic of Korea

${ }^{35}$ University of Rajasthan, Jaipur 302004, India

${ }^{36}$ Rice University, Houston, Texas 77251, USA

${ }^{37}$ Universidade de Sao Paulo, Sao Paulo, Brazil

${ }^{38}$ University of Science \& Technology of China, Hefei 230026, China

${ }^{39}$ Shandong University, Jinan, Shandong 250100, China

${ }^{40}$ Shanghai Institute of Applied Physics, Shanghai 201800, China

${ }^{41}$ SUBATECH, Nantes, France

${ }^{42}$ Texas AEMM University, College Station, Texas 7r843, USA

${ }^{43}$ University of Texas, Austin, Texas 78712, USA

${ }^{44}$ Tsinghua University, Beijing 100084, China

${ }^{45}$ United States Naval Academy, Annapolis, MD 21402, USA

${ }^{46}$ Valparaiso University, Valparaiso, Indiana 46383, USA

${ }^{47}$ Variable Energy Cyclotron Centre, Kolkata 700064, India

${ }^{48}$ Warsaw University of Technology, Warsaw, Poland

${ }^{49}$ University of Washington, Seattle, Washington 98195, USA

${ }^{50}$ Wayne State University, Detroit, Michigan 48201, USA

${ }^{51}$ Institute of Particle Physics, CCNU (HZNU), Wuhan 430079, China

${ }^{52}$ Yale University, New Haven, Connecticut 06520, USA

${ }^{53}$ University of Zagreb, Zagreb, HR-10002, Croatia

(Dated: November 14, 2009)

We report a measurement of the longitudinal double-spin asymmetry $A_{L L}$ and the differential cross section for inclusive $\pi^{0}$ production at midrapidity in polarized proton collisions at $\sqrt{s}=200 \mathrm{GeV}$. The cross section was measured over a transverse momentum range of $1<p_{T}<17 \mathrm{GeV} / c$ and found to be in good agreement with a next-to-leading order perturbative QCD calculation. The longitudinal double-spin asymmetry was measured in the range of $3.7<p_{T}<11 \mathrm{GeV} / c$ and excludes a maximal positive gluon polarization in the proton. The mean transverse momentum fraction of $\pi^{0}$ 's in their parent jets was found to be around 0.7 for electromagnetically triggered events.

PACS numbers: 13.87.Ce, 13.88.+e, 14.70.Dj, 12.38.Qk 
The spin structure of the nucleon is one of the fundamental and unresolved questions in Quantum Chromodynamics (QCD). Deep-inelastic scattering (DIS) experiments studying polarized leptons scattered off polarized nuclei have found the quark and anti-quark spin contributions to the overall spin of the nucleon to be small, at the level of $25 \%$ [1, 2, leading to increased interest in the spin contribution from gluons. DIS experiments have placed coarse constraints on the polarized gluon distribution function $\Delta g(x)$, based on the scale dependence of polarized structure functions [3, 4] and on recent semi-inclusive data [5, 6, 7]. Measurements using collisions of longitudinally polarized protons are attractive because they provide sensitivity to the polarized gluon spin distribution at leading order through quark-gluon and gluon-gluon scattering contributions to the cross section.

The sensitivity of inclusive hadron and jet production to the underlying gluon polarization in high-energy polarized proton collisions has been discussed in detail in Refs. [8, 9]. The theoretical framework in the context of next-to-leading order perturbative QCD (NLO pQCD) calculations is very well developed to constrain $\Delta g(x)$. The first global analysis of semi-inclusive and inclusive DIS data, as well as results obtained by the PHENIX [10] and STAR [11] experiments, placed a strong constraint on $\Delta g(x)$ in the gluon momentumfraction range of $0.05<x<0.2$, and suggested that the gluon spin contribution is not large in that range 12 . This conclusion was driven primarily by data on inclusive hadron and jet production in polarized proton collisions at $\sqrt{s}=200 \mathrm{GeV}$ at RHIC.

In this paper, we report on the measurement of the cross section and the longitudinal double-spin asymmetry $A_{L L}$ for inclusive $\pi^{0}$ production at midrapidity in polarized proton collisions at $\sqrt{s}=200 \mathrm{GeV}$ by the STAR experiment [13] at RHIC. The cross section is compared to a NLO pQCD calculation and the observed agreement provides an important basis to apply pQCD for the interpretation of $A_{L L}$. The asymmetry is defined as

$$
A_{L L} \equiv \frac{\sigma^{++}-\sigma^{+-}}{\sigma^{++}+\sigma^{+-}},
$$

where $\sigma^{++}$and $\sigma^{+-}$are the inclusive $\pi^{0}$ cross sections for equal $(++)$ and opposite $(+-)$ beam helicity configurations. The measured longitudinal double-spin asymmetry probes a gluon momentum fraction of approximately $0.03<x<0.3$, and is compared to NLO pQCD calculations. In addition, we present the mean transverse momentum fraction of $\pi^{0}$ 's in electromagnetically triggered jets. This measurement allows one to relate the spin asymmetry measurements performed with inclusive $\pi^{0}$ 's to those using reconstructed jets. It may also help to constrain fragmentation models.

The data for the analyses presented here were collected at STAR in 2005 using stored polarized $100 \mathrm{GeV}$ proton beams with an average luminosity of $6 \times 10^{30} \mathrm{~cm}^{-2} \mathrm{~s}^{-1}$. Longitudinal polarization of proton beams in the STAR interaction region (IR) was achieved by spin rotator magnets upstream and downstream of the IR that changed the proton spin orientation from its stable vertical direction to longitudinal 14. The helicities were alternated between successive proton bunches in one beam and pairs of successive proton bunches in the other beam. This allowed us to obtain all four helicity combinations of the colliding bunch pairs at the STAR IR in quick succession. Additional reduction of systematic uncertainties was achieved by periodically changing the helicity patterns of the stored beams. The polarization of each beam was measured several times per fill using Coulomb-Nuclear Interference (CNI) proton-carbon polarimeters 15, which were calibrated using a polarized hydrogen gas-jet target [16. The average RHIC beam polarizations in the 2005 run were $P_{1}=52 \pm 3 \%$ and $P_{2}=48 \pm 3 \%$. Non-longitudinal beam polarization components were continuously monitored with local polarimeters at STAR [17] and were found to be no larger than $9 \%$ in absolute magnitude.

The principal STAR detector subsystems for the measurements presented here were the Barrel Electromagnetic Calorimeter (BEMC) [18] and the Beam-Beam Counters (BBC) [17. In addition, the Time Projection Chamber (TPC) [19] was used for vertexing, for measuring the charged component in the reconstructed jets, and as a charged particle veto for the $\pi^{0}$ reconstruction. The BEMC is a lead-scintillator sampling calorimeter with a granularity of $\Delta \eta \times \Delta \varphi=0.05 \times 0.05 \mathrm{rad}$, where one such cell is referred to as a tower. It contains a shower maximum detector (BSMD) that consists of two layers of wire proportional counters with cathode strip readout, one in the azimuthal direction and one in the longitudinal direction, at a depth of about 5 radiation lengths in each calorimeter module, providing a segmentation of $0.007 \times 0.007 \mathrm{rad}$. For the 2005 running period, half of the BEMC was instrumented and operational, providing $2 \pi$ azimuthal coverage for $0<\eta<1$. The BBCs are composed of segmented scintillator rings, covering $3.3<|\eta|<5.0$ on both sides of the IR. The BBCs were used to trigger on collisions, to measure the helicitydependent relative luminosities, and to serve as local polarimeters. The TPC provided charged particle tracking inside a $0.5 \mathrm{~T}$ solenoidal magnetic field over the full range of azimuthal angles for $|\eta|<1.3$.

Proton-proton collisions in the STAR detector were identified by a minimum bias trigger (MB), defined as a coincidence of hits in both BBCs. The cross section for this trigger was $\sigma_{\mathrm{BBC}}=26.1 \pm 0.2$ (stat) \pm 1.8 (syst) mb, corresponding to $87 \pm 8 \%$ of the non-singly diffractive $p+p$ cross section at $\sqrt{s}=200 \mathrm{GeV}$ 20]. Rare hard scattering events were selected by two high-tower triggers, HT1 and $\mathrm{HT} 2$, that required a transverse energy deposition in a single BEMC tower above thresholds of 2.6 and $3.5 \mathrm{GeV}$, respectively, in addition to satisfying the $\mathrm{MB}$ condition.

A data sample with an integrated luminosity of $\mathcal{L}=$ $0.17 \mathrm{nb}^{-1}$ for MB, $0.16 \mathrm{pb}^{-1}$ for HT1, and $0.66 \mathrm{pb}^{-1}$ for HT2 triggers was analyzed for the inclusive cross section measurement. Data with an integrated luminosity of $0.4(2.0) \mathrm{pb}^{-1}$ of HT1 (HT2) triggers were used for the $A_{L L}$ determination. The event selection criteria for the asymmetry analysis were identical to those used in a previously published jet measurement [11. About $22 \%$ of HT1/HT2 triggered events also entered the jet $A_{L L}$ 
measurement [11], but represented a negligible fraction of the much larger inclusive jet data set. Therefore, the statistical correlation of the present $\pi^{0}$ and jet $A_{L L}$ measurements is negligible.

Neutral pions were reconstructed in the decay channel $\pi^{0} \rightarrow \gamma \gamma$ in an invariant mass analysis of pairs of neutral BEMC clusters, i.e., those that did not have a TPC track pointing to them, with a cut on the two-particle energy asymmetry of $\left|E_{1}-E_{2}\right| /\left(E_{1}+E_{2}\right) \leq 0.7$. The tower granularity was insufficient to resolve cluster pairs in HT1/HT2 data because of the small opening angle between daughter photons of pions that satisfied these triggers. Therefore, the BSMD clusters were used to determine the photon coordinates in those data. A fiducial volume cut on the detector pseudorapidity of $0.1<\eta<0.9$ was imposed. The reconstructed value of the pion pseudorapidity with respect to the vertex position was required to fall in the range $0<\eta<1$. The $\pi^{0}$ yield was extracted in $p_{T}$ bins by integrating the background-subtracted invariant mass distribution in a $p_{T}$-dependent window around the $\pi^{0}$ peak that corresponded to an approximately $\pm 3 \sigma$ range. The combinatorial background was determined using the event mixing method with a jet alignment correction [21, 22.

The cross section for $\pi^{0}$ production is given by

$$
E \frac{d^{3} \sigma}{d \mathbf{p}^{3}}=\frac{1}{2 \pi p_{T} \Delta p_{T} \Delta \eta} c \frac{N}{\mathcal{L}},
$$

where $\Delta p_{T}$ and $\Delta \eta$ are the bin widths in $p_{T}$ and pseudorapidity, $N$ is the $\pi^{0}$ yield in a bin, and $c$ is an overall correction factor that accounts for acceptance, reconstruction, and trigger efficiency in that bin, which was determined using a Monte Carlo simulation of $\pi^{0}$ 's passed through the GEANT [23] model of the STAR detector. Figure 1 shows the differential cross section for inclusive $\pi^{0}$ production. This analysis covered the pion transverse momentum range of $1<p_{T}<17 \mathrm{GeV} / c$, and data points were scaled to the bin centers using local exponential fits around each bin. The cross sections up to $4 \mathrm{GeV} / c$ were measured using MB triggered events; above 4 (7) $\mathrm{GeV} / c$ the entries were obtained from HT1 (HT2) triggers. The different trigger samples agreed within errors.

The dominant systematic uncertainty (25\% on average) of the measured cross section was due to a $5 \%$ uncertainty in the global energy scale of the BEMC. The other systematic uncertainties were related to yield extraction $(7 \%)$, reconstruction efficiency $(6 \%)$, and relative normalization of $\mathrm{HT} 1 / \mathrm{HT} 2$ and MB triggers (5\%). An additional uncertainty due to the limited quality of the electromagnetic shower simulation at low photon energies in our GEANT model was assigned to the cross section obtained from HT1/HT2 data $\left[15(2) \%\right.$ at $\left.p_{T}=4(7) \mathrm{GeV} / c\right]$.

In Fig. 1, the measured cross section is compared to a NLO pQCD calculation [8] performed using the CTEQ6M set of unpolarized parton distribution functions [26] and the DSS set of fragmentation functions 24]. In this calculation, the factorization and renormalization scales were identified with $p_{T}$ (solid curve), and were varied by a factor of two to estimate the impact of scale uncertainties (dashed curves). The DSS analysis included recent measurements of $\pi^{0}$ production at midrapidity by

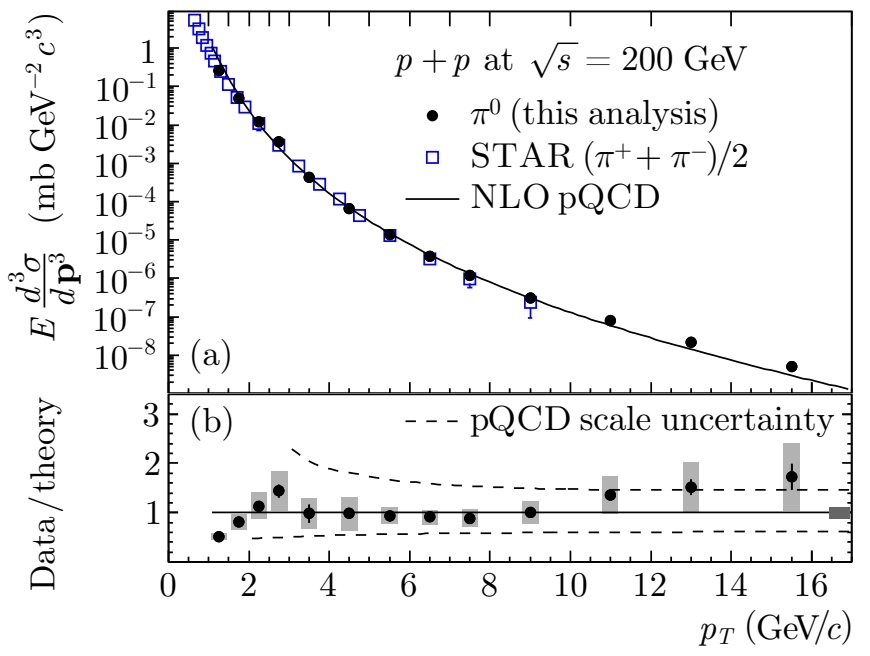

FIG. 1: (color online) (a) Cross section for inclusive $\pi^{0}$ production at midrapidity in $p+p$ collisions at $\sqrt{s}=200 \mathrm{GeV}$, compared to a NLO pQCD calculation 8 based on the DSS set of fragmentation functions 24], and to the STAR $\pi^{ \pm}$measurement 25]. (b) The ratio of measured cross section and the NLO pQCD calculation. The scale uncertainty is indicated by the dashed curves $\left(\mu=2 p_{T}, p_{T} / 2\right)$. The error bars are statistical and shaded bands are $p_{T}$-correlated systematic uncertainties. The normalization uncertainty is indicated by a shaded band around unity on the right-hand side.

PHENIX [10] and at forward rapidity by STAR 27. The NLO pQCD calculation shows, within errors, good agreement with our data in the fragmentation region $p_{T}>2 \mathrm{GeV} / c$. We also compare the cross section for $\pi^{0}$ production to the STAR $\pi^{ \pm}$measurement [25]. The $\pi^{0}$ and $\left(\pi^{+}+\pi^{-}\right) / 2$ cross sections are expected to be equal, and the two STAR measurements agree within statistical errors, in spite of using independent detector sub-systems.

The transverse momentum fraction carried by a high$p_{T} \pi^{0}$ in its parent jet, $z=p_{T}\left(\pi^{0}\right) / p_{T}$ (jet), was investigated by associating pions with jets found in the same event [28]. The $\pi^{0}$ sample, defined by the invariant mass window, contained $\approx 8 \%$ of combinatorial background. An association was made if the pion was within a cone of radius $R=\sqrt{(\Delta \eta)^{2}+(\Delta \varphi)^{2}}=0.4$ around the jet axis. The analysis was restricted to $0.4<\eta<0.6$ in the jet pseudorapidity, so that the reconstructed jets were fully contained in the BEMC acceptance. The transverse momentum of the jet was required to exceed $5 \mathrm{GeV} / c$. The jet was required to have a neutral energy fraction less than 0.95 , in order to minimize contributions from beam background to the reconstructed jet sample.

Figure 2(a) shows the mean value of $z$ as a function of pion $p_{T}$, combined for HT1 and HT2 triggers. The data points are plotted at the bin centers in pion $p_{T}$. The results were not corrected for detector effects, such as acceptance, efficiency, or resolution of the jet reconstruction. The systematic error band shown includes contributions from the uncertainty of the jet energy scale, the influence of the cut on minimum jet $p_{T}$, the contribution of events with $z>1$, and a variation of other analysis cuts.

The $\langle z\rangle$ of $\pi^{0}$ 's in electromagnetically triggered jets was 

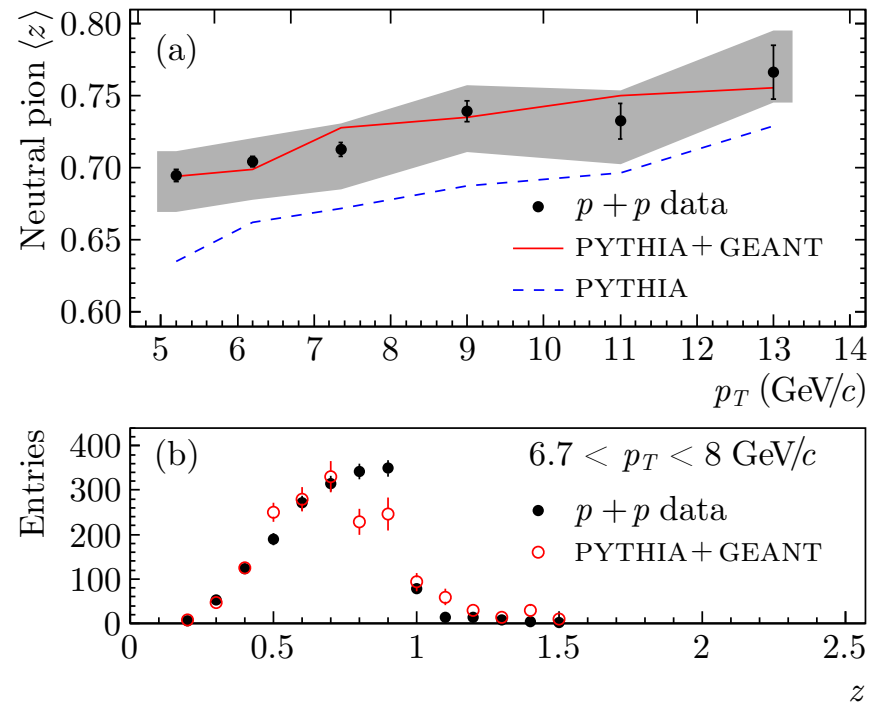

FIG. 2: (color online) (a) Mean transverse momentum fraction of $\pi^{0}$ 's in their associated jets, as a function of pion $p_{T}$, for electromagnetically triggered events. Systematic errors are shown by the shaded band around the data points. The curves are results from simulations with the PYTHIA event generator. The solid curve includes detector effects simulated by GEANT, while the dashed curve uses jet finding at the PYTHIA particle level. (b) The distribution of $z$ for one $p_{T}$ bin, compared to PYTHIA with a full detector response simulation.

found to be around 0.7 and to rise slightly with pion $p_{T}$, consistent with measurements of leading charged hadrons in jets in fixed-target experiments [29]. The results also compare well to recent theoretical calculations for charged pions [30, considering the increase of the measured pion momentum fraction due to energy not reconstructed in the jet. The expectations from a PYTHIA-based (version 6.205 [31] with 'CDF Tune A' settings [32]) Monte Carlo simulation are also shown. The $\langle z\rangle$ measured in jets found on the PYTHIA particle level, i.e., without any detector effects, is lower than in the data due to resolution effects and losses in the jet reconstruction, indicating the influence of the detector on the measurement. Results from a GEANT-based STAR detector simulation show good agreement with the data, demonstrating the reliability of the simulation framework used in the present analysis.

Figure 2(b) shows the distribution of $z$ for one of the bins in pion $p_{T}$ in comparison to PYTHIA with a GEANTbased detector simulation. To maximize the statistics in the simulation, the generator-level $\pi^{0}$ 's were used without requiring an explicit reconstruction. This led to a softening of the falling edge of the distribution at high $z$ in simulations, since a full GEANT simulation was used for the containing jets, but did not affect the mean of the distribution. A small fraction of the events had $z>1$, apparently corresponding to pions that carried more transverse momentum than their containing jet. This excess was caused by corrections applied during jet reconstruction, which in some cases led to an underestimation of the jet energy, and was well reproduced in simulations.

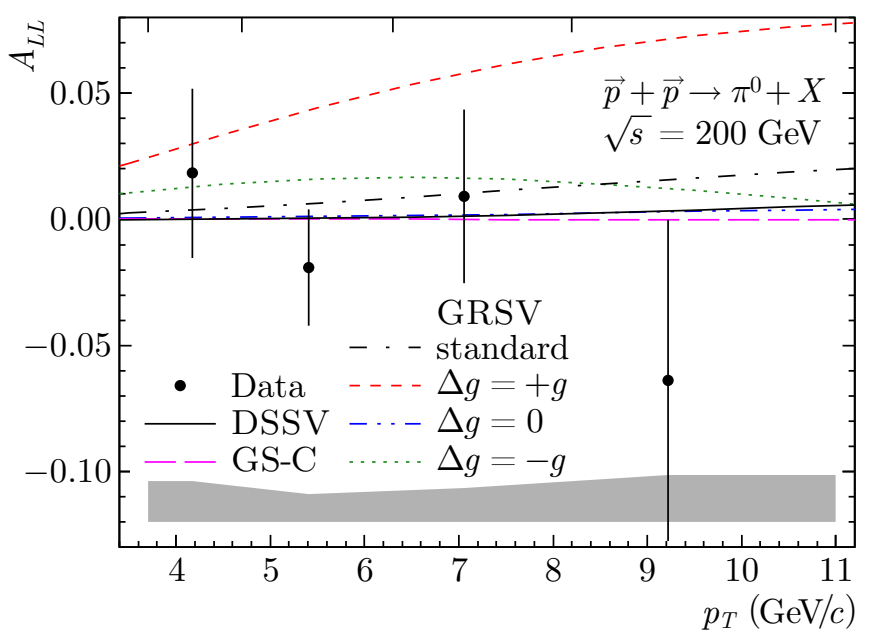

FIG. 3: (color online) Longitudinal double-spin asymmetry for inclusive $\pi^{0}$ production at midrapidity in $p+p$ collisions at $\sqrt{s}=200 \mathrm{GeV}$, compared to NLO pQCD calculations based on the gluon distributions from the GRSV [33], GS-C [34, and DSSV 12 global analyses. The systematic error (shaded band) does not include a $9.4 \%$ normalization uncertainty due to the beam polarization measurement.

The asymmetry [Eq. [1] ] was calculated as

$$
A_{L L}=\frac{1}{P_{1} P_{2}} \frac{\left(N^{++}-R N^{+-}\right)}{\left(N^{++}+R N^{+-}\right)},
$$

where $N^{++}$and $N^{+-}$are the $\pi^{0}$ yields in equal and opposite beam helicity configurations, respectively, and $R$ is the luminosity ratio for those two helicities. Typical values of $R$, measured with the BBCs to a statistical precision of $10^{-3}-10^{-4}$ per run, ranged from 0.85 to 1.2 , depending on fill and bunch pattern. Figure 3 shows the measured longitudinal double-spin asymmetry for $\pi^{0}$ production. The data points are plotted at the mean pion $p_{T}$ in each bin. The lowest- $p_{T}$ point at $4.17 \mathrm{GeV} / c$ was obtained from HT1 triggers only; other points are the HT1 and HT2 combined results.

The systematic errors shown in the figure include point-to-point contributions from $\pi^{0}$ yield extraction $\left[(4-14) \times 10^{-3}\right]$, invariant mass background subtraction $\left[(6-11) \times 10^{-3}\right]$, and remaining beam background [(1$\left.9) \times 10^{-3}\right]$, as well as $p_{T}$-correlated contributions from relative luminosity uncertainties $\left(9 \times 10^{-4}\right)$ and from nonlongitudinal spin components $\left(3 \times 10^{-4}\right)$. All of the errors above are absolute errors on the measured asymmetry. An evaluation of the effects of non-longitudinal components of the beam polarization was not possible due to the limited statistics of $\pi^{0}$ 's in data taken with transversely polarized beams. Instead, the largest value from the jet measurement [11] over the relevant momentum range was taken as an estimate of this systematic error. An overall normalization uncertainty of $9.4 \%$ due to the uncertainty in the RHIC CNI polarimeter calibration is not shown. Studies of parity-violating single spin asymmetries and randomized spin patterns showed no evidence of bunch-to-bunch or fill-to-fill systematics. 
In Fig. 3, the measured values for $A_{L L}$ are compared to NLO pQCD calculations [8] based on various sets of polarized gluon distribution functions. The DSSV curve [12] is the result of the first global analysis that includes semi-inclusive and inclusive DIS data, as well as results obtained by the PHENIX [10, and STAR [1] experiments. The GS-C curve [34] refers to a polarized gluon distribution function that has a large positive gluon polarization at low $x$, a node near $x \approx 0.1$, and a negative gluon polarization at large $x$. The GRSV standard curve is based on the best fit to DIS data [33, while the other GRSV curves show scenarios of extreme positive $(\Delta g=+g)$, extreme negative $(\Delta g=-g)$, and vanishing $(\Delta g=0)$ gluon polarization at the starting scale 9,33 . A maximal positive gluon polarization scenario, which has a total gluon spin contribution $\Delta G \equiv \int_{0}^{1} \Delta g(x) d x=1.26$ at an initial scale of $0.4 \mathrm{GeV}^{2}$ [33, 35], is excluded by our measurement at $98 \%$ confidence level, including systematic uncertainties. This is in agreement with the conclusions from the inclusive jet measurements by STAR [11, 28. and from the inclusive $\pi^{0}$ measurement by PHENIX [10] The data are consistent with all other gluon polarization scenarios, in particular with the DSSV case.

In summary, we report a measurement of the invariant cross section and the longitudinal double-spin asymmetry $A_{L L}$ for inclusive $\pi^{0}$ production at midrapidity with the STAR detector at RHIC. The cross section was determined for $1<p_{T}<17 \mathrm{GeV} / c$ and found to be in agreement with a NLO pQCD calculation based on the CTEQ6M parton distribution functions and the DSS fragmentation functions. This set of fragmentation functions was constrained by data that included measurements of $\pi^{0}$ production at midrapidity by PHENIX [10] and at forward rapidity by STAR 27. The mean transverse momentum fraction of $\pi^{0}$ 's in electromagnetically trig- gered jets was found to be approximately 0.7 and to rise slightly with pion $p_{T}$, in agreement with a PYTHIAbased Monte Carlo simulation that included detector effects. This measurement has the potential to contribute to future fragmentation function studies. The asymmetry $A_{L L}$ was measured in the hard scattering regime at $3.7<p_{T}<11 \mathrm{GeV} / c$ and found to be consistent with NLO pQCD calculations utilizing polarized quark and gluon distributions from inclusive and semi-inclusive DIS data and from polarized proton data. Our data exclude a maximal positive gluon polarization in the nucleon, in agreement with results obtained from inclusive jet production in polarized proton collisions by STAR [11, 28, while being a statistically independent measurement, subject to a different set of systematic uncertainties. With increasing integrated luminosity, the neutral pion channel has the potential to provide additional constraints on the gluon polarization in the polarized proton.

We thank the RHIC Operations Group and RCF at BNL, the NERSC Center at LBNL and the Open Science Grid consortium for providing resources and support. This work was supported in part by the Offices of NP and HEP within the U.S. DOE Office of Science, the U.S. NSF, the Sloan Foundation, the DFG cluster of excellence 'Origin and Structure of the Universe', CNRS/IN2P3, STFC and EPSRC of the United Kingdom, FAPESP $\mathrm{CNPq}$ of Brazil, Ministry of Ed. and Sci. of the Russian Federation, NNSFC, CAS, MoST, and MoE of China, GA and MSMT of the Czech Republic, FOM and NWO of the Netherlands, DAE, DST, and CSIR of India, Polish Ministry of Sci. and Higher Ed., Korea Research Foundation, Ministry of Sci., Ed. and Sports of the Rep. Of Croatia, Russian Ministry of Sci. and Tech, and RosAtom of Russia.
[1] J. Ashman et al. (EMC), Nucl. Phys. B328, 1 (1989).

[2] B. W. Filippone and X.-D. Ji, Adv. Nucl. Phys. 26, 1 (2001).

[3] B. Adeva et al. (SMC), Phys. Rev. D58, 112002 (1998).

[4] P. L. Anthony et al. (E155), Phys. Lett. B493, 19 (2000).

[5] A.Airapetian et al.(HERMES),Phys.Rev.Lett. 84,2584(2000).

[6] B. Adeva et al. (SMC), Phys. Rev. D70, 012002 (2004).

[7] E. S. Ageev et al. (COMPASS), Phys. Lett. B633, 25 (2006).

[8] B. Jäger, A. Schäfer, M. Stratmann, and W. Vogelsang, Phys. Rev. D67, 054005 (2003).

[9] B. Jäger, M. Stratmann, and W. Vogelsang, Phys. Rev. D70, 034010 (2004).

[10] A. Adare et al. (PHENIX), Phys. Rev. D76, 051106 (2007).

[11] B.I. Abelev et al. (STAR), Phys. Rev. Lett. 100,232003(2008).

[12] D. de Florian, R. Sassot, M. Stratmann, and W. Vogelsang, Phys. Rev. Lett. 101, 072001 (2008).

[13] K. H. Ackermann et al. (STAR), NIM A499, 624 (2003).

[14] I. Alekseev et al., NIM A499, 392 (2003).

[15] I. Nakagawa et al., AIP Conf. Proc. 915, 912 (2007).

[16] Y. I. Makdisi et al., AIP Conf. Proc. 915, 975 (2007).

[17] J. Kiryluk (STAR), SPIN 2004 Conf. Proc., 718 (2005).

[18] M. Beddo et al. (STAR), NIM A499, 725 (2003).
[19] M. Anderson et al. (STAR), NIM A499, 659 (2003).

[20] J. Adams et al. (STAR), Phys. Rev. Lett. 91, 172302 (2003).

[21] O. Grebenyuk, Ph.D. thesis, Utrecht University (2007).

[22] B. I. Abelev et al. (STAR), to be published.

[23] R. Brun, R. Hagelberg, M. Hansroul, and J. C. Lassalle (1978), CERN-DD-78-2-REV.

[24] D. de Florian, R. Sassot, and M. Stratmann, Phys. Rev. D75, 114010 (2007).

[25] J. Adams et al. (STAR), Phys. Lett. B637, 161 (2006).

[26] J. Pumplin et al., JHEP 07, 012 (2002).

[27] J. Adams et al. (STAR), Phys. Rev. Lett. 97, 152302 (2006).

[28] B. I. Abelev et al. (STAR), Phys. Rev. Lett. 97, 252001 (2006).

[29] G. Boca et al., Z. Phys. C49, 543 (1991).

[30] D. de Florian, Phys. Rev. D79, 114014 (2009).

[31] T. Sjöstrand,P.Edén, C.Friberg,L.Lönnblad, G.Miu,S.Mrenna, and E. Norrbin, Comput. Phys. Commun. 135, 238 (2001).

[32] R. Field and R. C. Group (CDF), hep-ph/0510198.

[33] M. Glück, E. Reya, M. Stratmann, and W. Vogelsang, Phys. Rev. D63, 094005 (2001).

[34] T. Gehrmann and W. J. Stirling, Phys. Rev. D53, 6100 (1996).

[35] M. Glück, E. Reya, and A. Vogt, Eur. Phys. J. C5, 461 (1998). 